\title{
Femoropoplital bypass versus endovascular treatment for long superficial femoral artery occlusion
}

\author{
Hesham Abu Greda, MD; Nehad Zaid, MD
}

\author{
Vascular Surgical Unit, Menoufiya University Hospitals, Menofya, Egypt.
}

\begin{abstract}
Aim: To evaluate the effectiveness of angioplasty and stenting versus above-knee bypass in the treatment of symptomatic long segment superficial femoral artery lesions.

Patients \& methods: 60 patients with symptoms of superficial femoral artery lesions $(\geq 10$ $\mathrm{cm}$ long) were enrolled into the study. Thirty patients were randomly assigned to percutaneous transluminal angioplasty (PTA) and primary stenting. Another 30 patients underwent bypass surgery. All patients in both groups were followed up at 1, 3, 6 and 12 months after either procedure.
\end{abstract}

Results: Patients who underwent endovascular treatment have got a primary patency rate $71.4 \%$ and a secondary patency rate $89.2 \%$. Patients who underwent fem-pop bypass have got a primary patency rate $77.7 \%$ and a secondary patency rate $81.4 \%$ at 12 months follow up. The primary \& secondary patency rates among both groups were not significantly different $(P$ $=0.754 \& 0.451$ respectively).

Conclusion: Endovascular treatment is an effective procedure as bypass surgery in the treatment of long superficial femoral artery lesions. It holds the advantages of short hospital stay and avoidances of surgery-related wound infection especially in diabetics.

Key words: Angioplasty, patency \& bypass surgery.

\section{Introduction:}

Management of symptoms of superficial femoral artery long lesions SFA poses a unique challenge for vascular surgeons worldwide. This is owing to the complex interplay of various factors including: the underlying pathology, anatomic defect, degree of ischemia, co-morbid conditions, functional status, previous ambulation and suitability of anatomy for successful revascularization.

There are two widely available treatment options for lower limb revascularization (1) bypass surgery and (2) endovascular treatment. The goals of both modalities are relieving pain, promoting wound healing, and preserving the best limb function, with the least cardiovascular risks.

Bypass surgery had been the treatment of choice for long SFA lesions but with recent advances in endovascular tools many controversies had been aroused about which of the two modalities should be the first line for treatment of these lesions. ${ }^{1}$

\section{Patients and methods:}

This prospective study was conducted on 60 patients suffering from symptomatic chronic lower limb ischemia due to $\geq 10 \mathrm{~cm}$ long superficial femoral artery lesions. Patients were randomized into 2 treatment groups. The first group; included patients who underwent fem-pop bypass graft. The second group; included patients who underwent PTA with primary stenting for the SFA lesions.

All patients underwent duplex scanning and CT angiography. All angiograms had been reviewed by a vascular surgeon and an interventional radiologist. All patients who participated in the study had at least one patent crural artery. Ankle brachial index was recorded. Also laboratory tests were done including the kidney \& liver functions, coagulation profiles and complete blood picture. 
The bypass procedure was achieved using an ePTFE (expanded polytetrafluoroethylene) graft 6-8 $\mathrm{mm}$ according to size of the native artery. Postoperative low molecular weight heparin and intravenous antibiotic was maintained for three days.

Patients in the endovascular group: were accessed percutaneously in the contralateral common femoral artery via standard Seldinger technique and were fully anticoagulated with heparin (100 units $/ \mathrm{kg}$ ) during the procedure. Transluminal or subintimal plan was used to cross occlusive lesions with pre-dilatation angioplasty of the lesion to be treated. Stent deployment was accomplished with selfexpandable nitinol stents sized according to vessel lumen. Following stent placement; patients were immediately started on aspirin ( $150 \mathrm{mg} /$ day) and clopidogrel ( $75 \mathrm{mg} /$ day) for a minimum of 3 months then were maintained on aspirin only.

After discharge, all patients in both groups were followed up on out-patient basis at 1, 3, 6 and 12 months. Patient assessment was essentially focused on: lower limb clinical examination, determination of Ankle Brachial Index (ABI) and color flow Doppler ultrasound.

Statistical analysis:

Continuous variables were presented as mean and standard deviation; Categoric variables were presented as counts and percentages. Differences of categoric variables were assessed by the two-tailed Fisher Exact test. Differences between continuous variables were assessed by twotailed unpaired t-test. Primary patency rates were compared between the surgical bypass and endovascular treatment groups by using the log-rank test and are presented as KaplanMeier curves. AP-value $<0.05$ was considered statistically significant.

\section{Results:}

Between January 2013 and January 2014, 30 patients were treated percutaneously with angioplasty and primary stenting and 30 patients were treated surgically with femoral to above-knee popliteal artery bypass.

The analysis was based on 60 patients consisting of 17 females \& 43 males with mean age $55.6 \pm 9$ y (range from $40-71$ years). The gender and age differences between both groups were not significant. These data are shown in Table (1).

The distal perfusion in both groups presented with $\mathrm{ABI}$ was $0.45 \pm 0.12$ in the bypass group versus $0.50 \pm 0.13$ in the endovascular group. The differences in pretreatment clinical presentation and average ABI in both groups was not significant. This is shown in Table (2).

By following the TASC II grading system for femoral-popliteal lesions, each limb in both treatment groups was assigned a TASC II classification as shown in Table (3). There was no significant difference in TASC II classification between the two treatment groups. The demography of arterial lesions and number of patent crural arteries in both groups is shown in Table (4).

Above knee femoro-popliteal bypass, using ePTFE graft was successfully performed in all treated limbs in the surgical bypass group. The mean diameter of the used grafts was 6.6 $\mathrm{mm}$ (range 6 to $8 \mathrm{~mm}$ ). The mean duration of the operation was $190 \pm 29.3$ minutes.

In the endovascular treatment group, contralateral femoral artery puncture was successfully performed in all patients. Transluminal crossing of target lesion was performed in 17 patients while subintimal crossing was performed in 13 patients. Stents were successfully deployed in all patients. The mean duration of the procedure was 56 \pm 13.9 minutes.

Length of hospital stay was analyzed for both groups, the mean hospital stay was 3.1 \pm 1.21 days for the endovascular treatment group, and $9.8 \pm 3.45$ days for the surgical bypass group, This difference proved to be significant $(\mathrm{P}=0.001)$.

Marked improvement of the pain free walking distance and relief of rest pain was recorded in the early postoperative period. Average ankle brachial index was improved by $0.37 \pm 0.08$ in bypass group and by 0.34 \pm 0.07 in endovascular group. This difference between the two groups was not significant $(\mathrm{P}=0.12)$. 


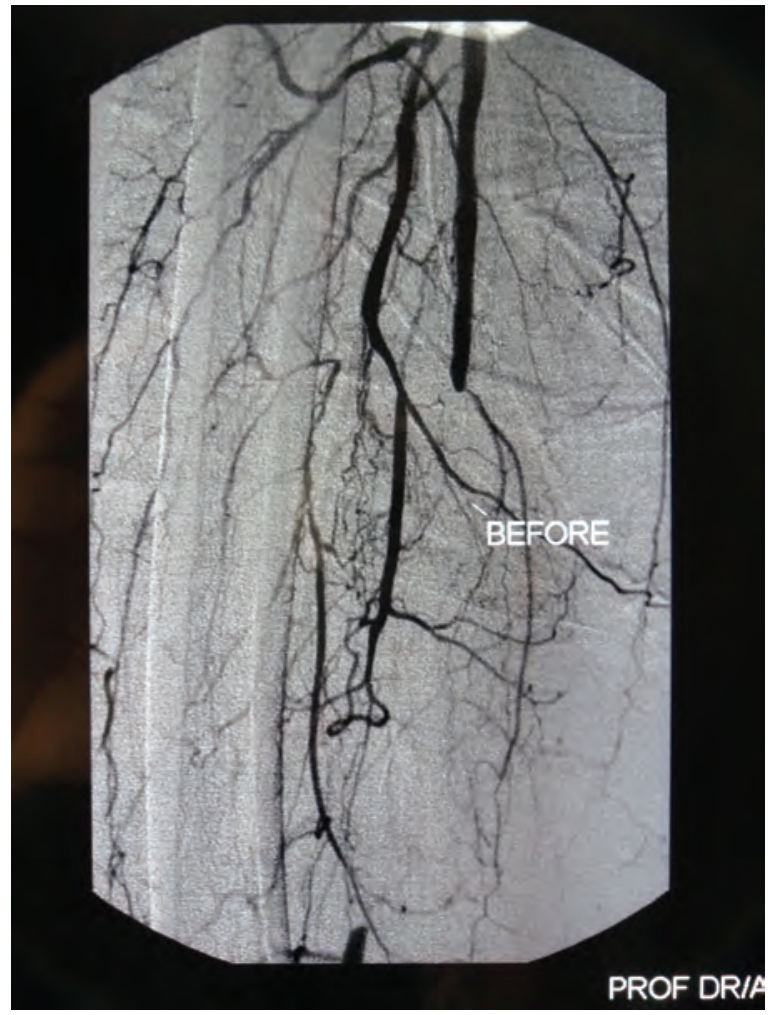

Figure (1): Superfacial femoral artery.

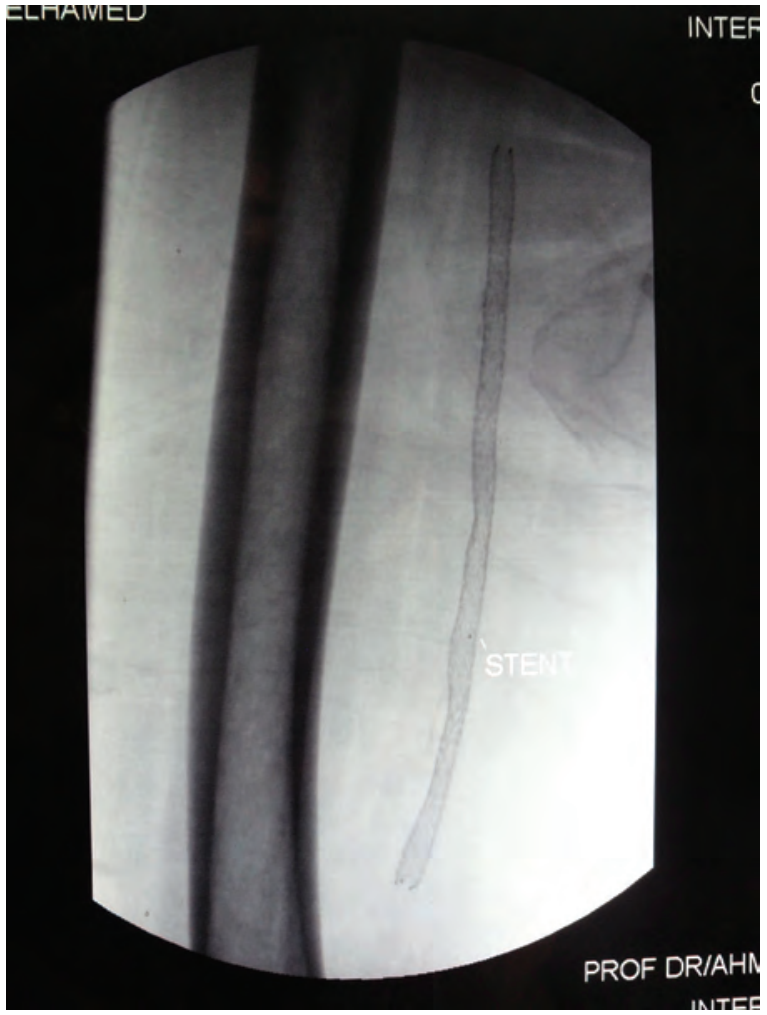

Figure (2): Occlusion stent placement.

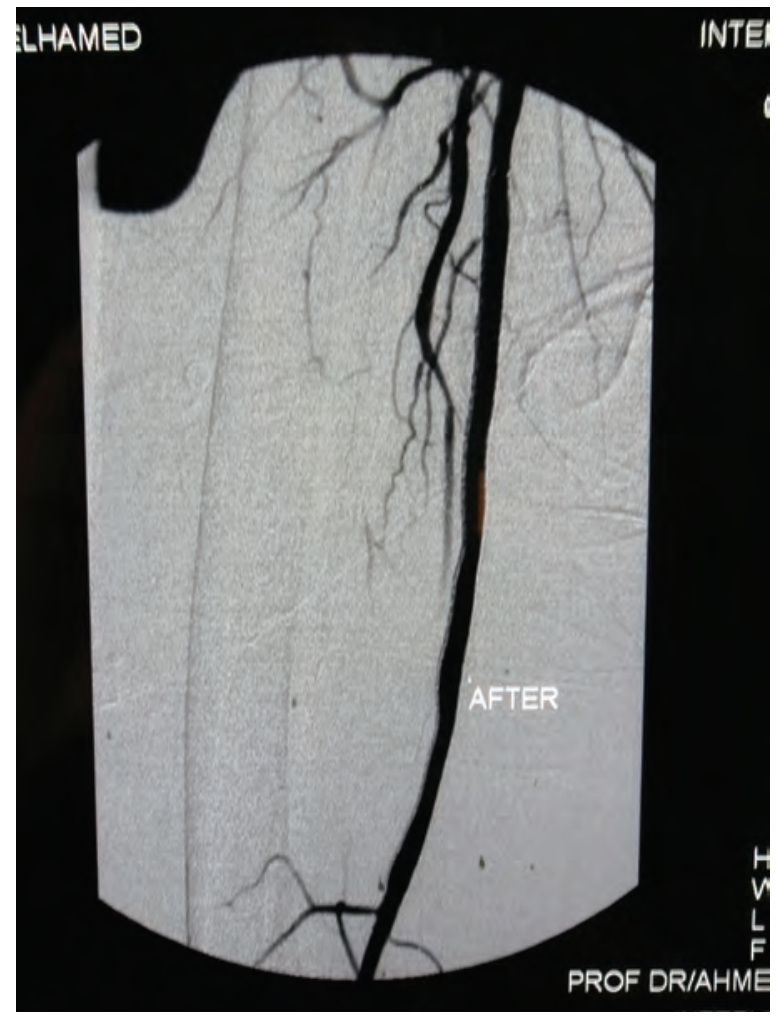

Figure (3): Refilling after stent placement.

Mean follow-up duration was 18 months (range from 12-24 months) for both treatment groups. Complete foot wound healing was achieved after $55.3 \pm 12.6$ (range: $35-75$ days) in bypass group versus $60.6 \pm 14.1$ (range:
40-95 days) in the endovascular group. However; this difference was not significant $(\mathrm{P}=0.13)$.

In the surgical bypass group; early nonthrombotic complications were observed in 


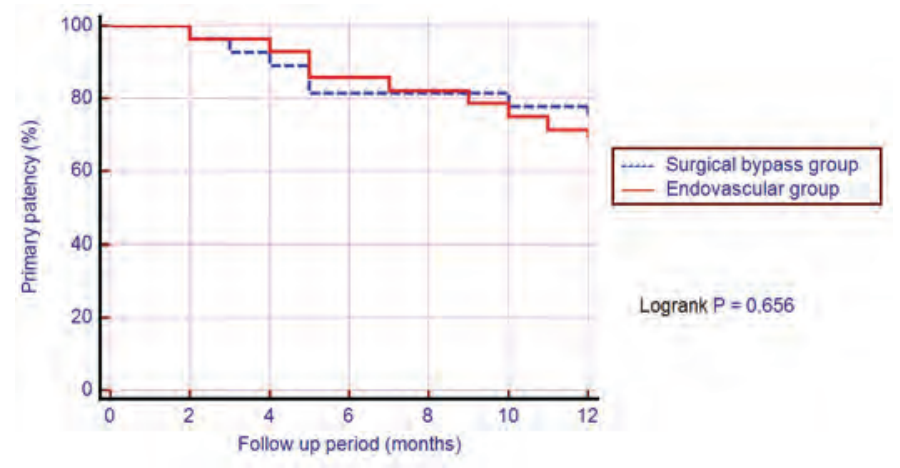

Figure (4): Primary patency rates in both groups.

Table 1. Patients characteristics in both treatment groups.

\begin{tabular}{|l|l|l|l|}
\hline & $\begin{array}{c}\text { Bypass group } \\
(\mathrm{n}=30)\end{array}$ & $\begin{array}{c}\text { Endovasc group } \\
(\mathrm{n}=30)\end{array}$ & $\begin{array}{c}\mathrm{P} \\
\text { value }\end{array}$ \\
\hline Male / female & $21 / 9$ & $22 / 8$ & 1.00 \\
\hline $\begin{array}{l}\text { Mean age } \text { ( SD } \\
\text { range in years) }\end{array}$ & $\begin{array}{l}55.6 \pm 9 \mathrm{y} \\
(40-71)\end{array}$ & $\begin{array}{l}56.8 \\
\pm 9.2 \\
(45-70)\end{array}$ & 0.61 \\
\hline Hypertension & $18(60 \%)$ & $17(56.6 \%)$ & 1.00 \\
\hline Hyperlipidemia & $19(63.3 \%)$ & $16(53.3 \%)$ & 0.6 \\
\hline Diabetes & $18(60 \%)$ & $22(73.3 \%)$ & 0.41 \\
\hline Myocardial infarction & $3(10 \%)$ & $2(6.6 \%)$ & 1.00 \\
\hline Cerebral stroke & $1(3.3 \%)$ & none & 1.00 \\
\hline Smoking & $11(36.6 \%)$ & $10(33.3 \%)$ & 1.00 \\
\hline
\end{tabular}

Table 2. Clinical features in both groups.

\begin{tabular}{|l|l|l|l|}
\hline Clinical presentation & Bypass group & Endovasc group & P value \\
\hline Severe intermittent caludication (I.C) & $5(16.6 \%)$ & $7(23.3 \%)$ & 0.74 \\
\hline Rest pain & $11(36.6 \%)$ & $10(33.3 \%)$ & 1.00 \\
\hline Tissue loss & $14(46.6 \%)$ & $13(43.3 \%)$ & 1.00 \\
\hline average Ankle brachial index \pm SD & $0.45 \pm 0.12$ & $0.50 \pm 0.13$ & 0.12 \\
\hline
\end{tabular}

Table 3. TASC II grading of each limb in both treatment groups.

\begin{tabular}{|l|l|l|l|}
\hline \multicolumn{1}{|c|}{ TASC II grade } & Surgical bypass group $(n=30)$ & Endovascular group $(n=30)$ & P value \\
\hline B & 14 & 12 & 0.79 \\
\hline C & 16 & 18 & 0.79 \\
\hline
\end{tabular}

$8 / 30$ patients $(26.7 \%)$. Surgical site infection and wound dehiscence were managed nonoperatively except in two patients where secondary suture was done few days later.
In the endovascular group; immediate procedure-related and early post-operative, non-thrombotic complications were observed in $4 / 30(13 \%)$ patients. In two patients, 
Table 4: Arterial lesions and number of patent crural arteries.

\begin{tabular}{|c|c|c|c|}
\hline & Bypass group & Endovascular group & P value \\
\hline $\begin{array}{l}\text { Length of lesion in } \mathrm{cm} \pm \mathrm{SD} \\
\text { (range) }\end{array}$ & $\begin{array}{l}14.4 \mathrm{~cm} \pm 2.17 \\
(10-18 \mathrm{~cm})\end{array}$ & $\begin{array}{l}15.2 \mathrm{~cm} \pm 1.95 \\
(11-18 \mathrm{~cm})\end{array}$ & 0.13 \\
\hline \multicolumn{4}{|c|}{ Number of patent crural arteries } \\
\hline 1 & $7(23.3 \%)$ & $9(30 \%)$ & 0.77 \\
\hline 2 & $17(56.6 \%)$ & $16(53.3 \%)$ & 1.00 \\
\hline 3 & $6(20 \%)$ & $5(16.6 \%)$ & 1.00 \\
\hline
\end{tabular}

Table 5: Patency rates between the two groups.

\begin{tabular}{|c|c|c|c|}
\hline & Surgical bypass group & Endovascular treatment group & P value \\
\hline \multicolumn{4}{|c|}{ Primary patency } \\
\hline 1 month & $28 / 28(100 \%)$ & $30 / 30(100 \%)$ & \\
\hline 3 months & $26 / 28(92.8 \%)$ & $29 / 30(96.6 \%)$ & 0.60 \\
\hline 6 months & $23 / 28(82.1 \%)$ & $25 / 29(86.2 \%)$ & 0.72 \\
\hline 12 months & $21 / 27(77.7 \%)$ & $20 / 28(71.4 \%)$ & 0.75 \\
\hline \multicolumn{4}{|c|}{ Secondary patency } \\
\hline at 12 months & $22 / 27(81.4 \%)$ & $25 / 28(89.2 \%)$ & 0.46 \\
\hline
\end{tabular}

arterial wall dissection did occur in the SFA during passage of a guide-wire where the stent was used to exclude the dissection along with the atherosclerotic lesion. Another two patients had a small groin hematoma that were managed conservatively.

In the bypass group; 30 days mortality rate was $6.66 \%$ (2/30 patients), another patient died during the follow up period. In the endovascular group, 30 days mortality was $0 \%$, but two patients mortality was faced during follow up period. Mortality faced in both groups was diagnosed as massive myocardial infarction in 4 cases and cerebral stroke in one case. All deaths were unrelated to the procedure performed.

In the surgical bypass group; two patients underwent above knee amputation of the target limb owing to progressive tissue loss due to graft thrombosis within 6 months after the operation. In the endovascular treatment group, there was no major target limb amputation, with significance level $(\mathrm{P}=0.23)$.

Cumulative primary patency rates calculated at follow-up at 1, 3, 6 and 12 months in surgical bypass group were $100 \%$, $92.8 \%, 82.1 \%$ and $77.7 \%$ respectively; and in the endovascular treatment group it were $100 \%, 96.6 \%, 86.2 \%$ and $71.4 \%$ respectively. Secondary patency rate at the 12 -month follow-up was $81.4 \%$ in surgical bypass group and $89.2 \%$ in the endovascular treatment group. The differences in primary and secondary patency rates between the two treatment groups and their significance level are shown in Table (5) and presented by Figure (1).

\section{Discussion:}

The choice of treatment of long SFA occlusion is a matter of controversy over the last couple of decades especially with the great advances of endovascular techniques \& resources.

In the present study; we did try to evaluate the effectiveness of endovascular treatment versus the convention surgical bypass for long SFA occlusion. The main goal of our study was focused on the patency of either treatment modality.

The primary patency rate calculated at 12-month follow-up was better in bypass graft patients than those in endovascular group $(77.7 \%$ versus $71.4 \%)$. However; the difference in the primary patency rate 
between either groups was not significant. On the other hand; the secondary patency rate at 12-month follow-up was better in endovascular patients than those in the bypass graft one (89.6\% versus $81.4 \%$ ). Also; the difference between either groups was not significant. These findings did not come in agreement with the findings of patency rates disagree with many previous studies that had compared bypass to angioplasty and stenting in long SFA lesions. ${ }^{2,3}$

Stephen and Albert in 2003, have reported that treatment of long SFA lesions with angioplasty and primary stenting resulted in lower long-term patency rate than those with surgery, and it might be an acceptable alternative in selected patients with critical ischemia but bypass remains the procedure of choice. ${ }^{2}$ Also; Y1lmaz et al. in 2003 have reported that despite recent advances in endovascular treatment and even with the use of subintimal angioplasty, femoropopliteal bypass surgery still offers the best patency in the management of long SFA occlusions. ${ }^{3}$ Furthermore; Schlager et al. in 2005 have also reported that long segment stenting has a high rate of re-stenosis with 12-months primary patency of $54 \%$ and recommended it for only poor surgical patients with significant cardiovascular comorbidity. ${ }^{4}$

These differences in the patency rates between the present study and the previously mentioned studies may be explained by the absence of randomization in these studies or the advances in the endovascular tools as the use of nitinol stents which had higher patency and lower complication rate than stainless steel stents. This sight has reported by Nakagawa et al. in 2009; who compared the use of two stents in the management of long SFA lesions. ${ }^{5}$ Similar believe was achieved earlier by Ihnat et al.in 2008. ${ }^{6}$ They have reported that early experiences with stainless-steel stents showed no benefit over angioplasty alone but comparing superficial femoral artery angioplasty alone with nitinol stenting have shown a reduced incidence of restenosis with primary stenting.

Most of surgeons believe that bypass surgery is the best treatment for long SFA lesions and their decisions are based on the TASC II guidelines which had recommended bypass surgery for these lesions, because percutaneous treatments are considered less effective. ${ }^{7}$ However, since the release of these guidelines till now many studies had been conducted to re-evaluate the effectiveness of both modalities in long SFA lesions.

Many studies have compared bypass surgery with endovascular treatment and concluded results similar to our study, Dosluoglu et al., had compared stenting versus above knee polytetrafluoroethylene bypass for long SFA lesions and had reported that the 12- and 24-month primary patency was $83 \%$ and $80 \%$ for stenting group; versus $81 \%$ and $75 \%$ for the bypass group, and they had reported that secondary patency was significantly better in stenting group than bypass group. ${ }^{8}$

In addition, McQuade et al., had reported that the stent group demonstrated a primary patency of $72 \%$ and secondary patency of $83 \%$ at 12 months while the surgical group demonstrated a primary patency $77 \%$ and a secondary patency of $86 \%$ at 12 months with no statistical difference between the two groups with respect to primary or secondary patency. ${ }^{9}$ Similar results were reported by Scali et al., who had concluded that equivalent outcomes of endovascular and open surgical revascularization of the SFA could be achieved regardless of indication or TASC classification. ${ }^{10}$

Many authors have evaluated the use of angioplasty and stenting in the treatment of long SFA lesions, Ferreira et al. in 2007 have reported that primary and primary-assisted patency rates at $1,2 \& 3$ years were $90 \%, 78 \%$, $74 \%$; and $96 \%$, 90\%, 90\%, respectively and considered it as a good outcome considering length of lesions. ${ }^{11}$ In addition, Bosiers et al., had reported that long segment stenting of SFA had a low incidence of more than $50 \%$ restenosis and low fracture rate at 12 months and suggested that nitinol stent offers a safe and acceptably efficacious means of treating SFA lesions in symptomatic subjects with PAD. 12

Taneja et al., had reported that bare nitinol 
stent enabled recanalization of long segment lesions of superficial femoral artery with favorable midterm outcome and acceptable limb salvage rates but repeated interventions were required. ${ }^{13} \mathrm{Hu}$ et al., had also concluded that nitinol self-expanding stent implantation seems to be a good choice for patients with long SFA occlusions with good short and midterm patency results, but more observations are needed to assess its long-term efficiency. ${ }^{14}$ Similar results were reported by Connors et al., who had concluded that percutaneous treatment of long femoral artery stenoses and occlusions could achieve high long-term patency rates and recommended out-patient surveillance and re-intervention for recurrent symptoms and restenosis for the durability of long lesions. 15

Furthermore; other studies considered the use of covered stents instead of bare stents in the treatment of long SFA lesions, Kedora et al., \& Farraj et al., had concluded that percutaneous treatment with a stent graft in long SFA lesions had primary and primary assisted patency rates at 1 year comparable to above knee femoro-popliteal bypass using PTFE grafts. ${ }^{16,17}$ McQuade et al., also had reported that management of superficial femoral artery occlusive disease with percutaneous stent-grafts exhibits similar primary patency at 24-month follow-up when compared with conventional femoralpopliteal artery bypass grafting with synthetic conduit even in long SFA lesions. ${ }^{18}$

Beside patency rates in either treatment modality we did observe the procedure time consuming, the hospital stay and the wound complications. In all these observations endovascular procedures was superior to the bypass surgery.

In the present study, both bypass surgery and the endovascular procedure had comparable results with no significant difference regarding improvement of clinical presentation, improvement of average ankle brachial index, wound healing, mortality and patency rates. Although all patients in this study were fit for surgery and endovascular procedure, the later can be used also in treatment of poor surgical candidates.
Surgical site infection and wound complication are morbidities associating surgery and their absence gives advantage to endovascular procedures. Endovascular interventions also have the advantages of being minimal invasive procedures, can be performed under local anesthetic, repeatable, can be performed safely in fragile patients having high cardiovascular risks and they do not interfere with any bypass surgery if indicated later on. Therefore, endovascular treatment should be the first choice for management of long superficial femoral artery occlusive disease and bypass surgery could be considered only after failure of endovascular interventions.

\section{Conclusion:}

Endovascular treatment has similar results with regard to wound healing, mortality and patency rates when compared with bypass surgery in lower limb ischemia secondary to long superficial femoral artery lesions. It also has a shorter procedure time and hospital stay than surgery with lower incidence of complications so endovascular treatment should be the first choice for management of these lesions.

\section{Reference:}

1- White JV: Lower Extremity Arterial Disease: General Considerations, Ch 103 in: Cronenwett JL, Johnston KW, Cambria RP, Gloviczki P et al., eds. Rutherford's Vascular Surgery. $7^{\text {th }}$ ed. 2010.Elsevier, Inc.

2- Stephen WK, Albert CW: Angioplasty and primary stenting of high-grade, longsegment superficial femoral artery disease: Is it worthwhile? Ann Vasc Surg 2003; 17(4): 430-437.

3- Y1lmaz S, Sindel T, Yegin A, et al: Subintimal angioplasty of long superficial femoral artery occlusions. J Vasc Interv Radiol 2003; 14: 997-1010.

4- Schlager O, Dick P, Sabeti S, et al: Longsegment SFA stenting: The dark sides. In: Stent restenosis, clinical deterioration, and stent fractures. J Endovasc Ther 2005; 12: 676-684.

5- Nakagawa Y, Yajima J, Oikawa Y, et al: Clinical outcomes after percutaneous peripheral intervention for chronic total 
occlusion of superficial femoral arteries: Comparison between self-expandable nitinol stent and stainless steel stent. J Cardiology 2009; 53: 417- 421.

6- Ihnat DM, Duong ST, Taylor ZC, et al: Contemporary outcomes after superficial femoral artery angioplasty and stenting: The influence of TASC classification and run-off score. J Vasc Surg 2008; 47: 967-974.

7- Norgren L, Hiatt WR, Dormandy JA, et al: Intersociety consensus for the management of peripheral arterial disease TASC II. Eur J Vasc Endovasc Surg 2007; 33: S1-S75.

8- DosluogluHH, Cherr GS, Lall P, et al: Stenting vs above knee polytetrafluoroethylene bypass for Trans Atlantic Inter-Society Consensus-II $\mathrm{C}$ and D superficial femoral artery disease. $J$ Vasc Surg 2008; 48: (5) 1166-1174.

9- McQuade K, Gable D, Hohman S, et al: Randomized comparison of nitinol selfexpanding stent vs prosthetic femoralpopliteal bypass in the treatment of superficial femoral artery occlusive disease. J Vasc Surg 2009; 49: (1) 109-116.

10- Scali TC, Bjerke AA and Stone DH, et al: Long-term results of open and endovascular revascularization of superficial femoral artery occlusive disease. J Vasc Surg 2011; 54: 714-721.

11- Ferreira M, Lanziotti L, Monteiro M, et al: Superficial femoral artery recanalization with self-expanding nitinol stents: long-term follow-up results. Eur J Vasc Endovasc Surg 2007; 34: 702-708.

12- Bosiers M, Torsello G, Gissler HM, et al: Nitinol stent implantation in long superficial femoral artery lesions: 12-month results of the DURABILITY I study. J Endovasc Ther
2009; 16(3): 261-269.

13- Taneja M, Taya KH, Dewana A, et al: Bare nitinol stent enabled recanalization of longsegment, chronic total occlusion of superficial femoral and adjacent proximal popliteal artery in diabetic patients presenting with critical limb ischemia. Cardiovasc Revasc Medicine 2010; 11: 232-235.

14- Hu H, Zhang H, He Y, et al: Endovascular nitinol stenting for long occlusive disease of the superficial femoral artery in critical limb ischemia: A single-center, mid-term result. Ann Vasc Surg 2011; 25(2): 210-216.

15- Connors G, Todoran TM, Engelson BA, et al: Percutaneous revascularization of long femoral artery lesions for claudication: Patency over 2.5 years and impact of systematic surveillance. Catheterization and Cardiovascular Interventions 2011; 77: 1055-1062.

16- Kedora J, Hohmann S, Garrett W, et al: Randomized comparison of percutaneous Viabahn stent grafts vs prosthetic femoralpopliteal bypass in the treatment of superficial femoral arterial occlusive disease. $J$ Vasc Surg 2007; 45: 10-16.

17- Farraj N, Srivastava A, Pershad A: Oneyear outcomes for recanalization of long superficial femoral artery chronic total occlusions with the Viabahn stent graft. $J$ Invasive Cardiol 2009; 21(6): 278-281.

18- McQuade K, Gable D, Hohman S, et al: Randomized comparison of nitinol selfexpanding stent vs prosthetic femoralpopliteal bypass in the treatment of superficial femoral artery occlusive disease. J Vasc Surg 2009; 49: 109-116. 